\title{
Kerov's interlacing sequences and random matrices
}

\author{
Alexey Bufetov*
}

\begin{abstract}
To a $N \times N$ real symmetric matrix Kerov assigns a piecewise linear function whose local minima are the eigenvalues of this matrix and whose local maxima are the eigenvalues of its $(N-1) \times(N-1)$ submatrix. We study the scaling limit of Kerov's piecewise linear functions for Wigner and Wishart matrices. For Wigner matrices the scaling limit is given by the Verhik-Kerov-Logan-Shepp curve which is known from asymptotic representation theory. For Wishart matrices the scaling limit is also explicitly found, and we explain its relation to the Marchenko-Pastur limit spectral law.
\end{abstract}

\section{Introduction}

Consider two sequences of real numbers $\left\{x_{i}\right\}_{i=1}^{n},\left\{y_{j}\right\}_{j=1}^{n-1}$ such that

$$
x_{1} \geq y_{1} \geq x_{2} \geq \cdots \geq x_{n-1} \geq y_{n-1} \geq x_{n} .
$$

We say that the sequences $\left\{x_{i}\right\}_{i=1}^{n},\left\{y_{j}\right\}_{j=1}^{n-1}$ interlace. Define

$$
z_{0}=\sum_{i=1}^{n} x_{i}-\sum_{j=1}^{n-1} y_{j} .
$$

Following Kerov (see [5]) we define a rectangular Young diagram $w^{\left\{x_{i}\right\},\left\{y_{j}\right\}}(x)$ which is uniquely determined by the following conditions (see Figure 1):

1) $w^{\left\{x_{i}\right\},\left\{y_{j}\right\}}(x): \mathbb{R} \rightarrow \mathbb{R}$ is a continuous piecewise linear function, and $\frac{\partial}{\partial x} w^{\left\{x_{i}\right\},\left\{y_{j}\right\}}(x)= \pm 1$, except for finitely many points, which are exactly the local extrema of the function $w^{\left\{x_{i}\right\},\left\{y_{j}\right\}}(x)$.

2) $\left\{x_{i}\right\}_{i=1}^{n}$ are local minima of $w^{\left\{x_{i}\right\},\left\{y_{j}\right\}}(x),\left\{y_{j}\right\}_{j=1}^{n-1}$ are local maxima of $w^{\left\{x_{i}\right\},\left\{y_{j}\right\}}(x)$, and there are no other local extrema.

3) $w^{\left\{x_{i}\right\},\left\{y_{j}\right\}}(x)=\left|x-z_{0}\right|$ when $|x|$ is large enough.

Let $S$ be a $N \times N$ real symmetric matrix. By $\hat{S}$ we denote its $(N-$ $1) \times(N-1)$ submatrix; it is obtained from $S$ by removing the $N$ th row

*Institute for Information Transmission Problems, Independent University of Moscow and Higher School of Economics, e-mail: alexey.bufetov@gmail.com 


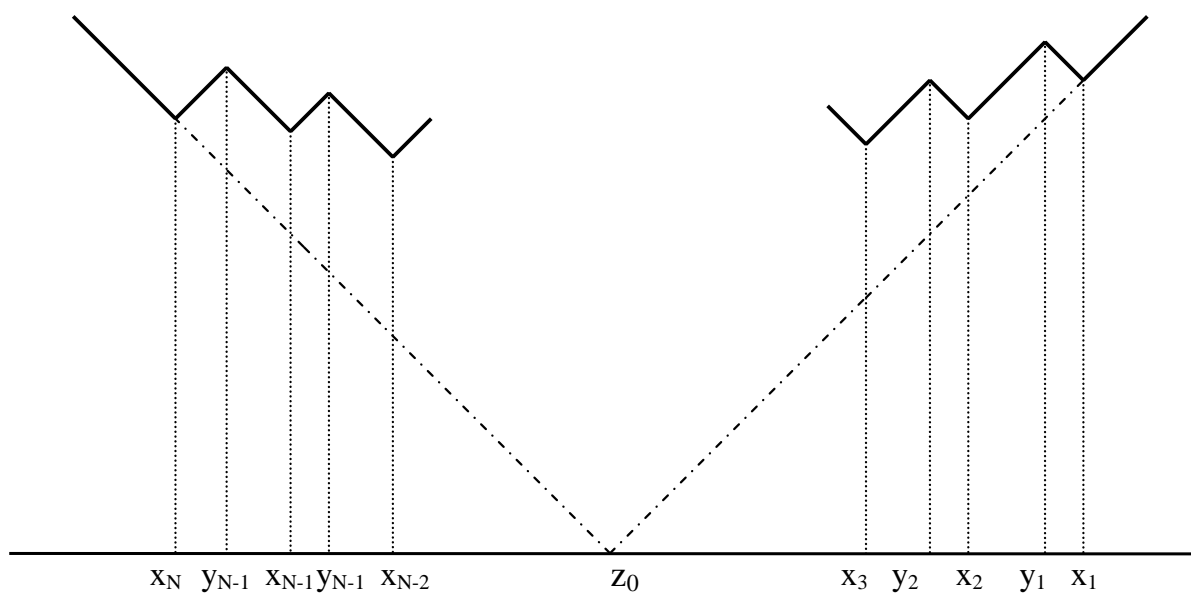

Figure 1: A part of a rectangular Young diagram

and column. It is well-known that the eigenvalues of $S$ and $\hat{S}$ interlace (see, e.g, [3, p.185]). Thus, to any symmetric matrix we can assign a rectangular Young diagram built from the eigenvalues of $S$ and $\hat{S}$.

Let $\left\{Z_{i j}\right\}_{i, j=1}^{\infty}$ be a family of independent and identically distributed zero-mean, real-valued random variables such that $\mathbf{E} Z_{11}^{2}=1$ and

$$
\mathbf{E}\left|Z_{11}\right|^{k}<\infty, \quad \text { for all } k=1,2,3, \ldots
$$

A symmetric $N \times N$ matrix $X_{N}$ with entries

$$
X_{N}(i, j)=X_{N}(j, i)=Z_{i j}, \quad \text { for } i \leq j,
$$

is called a Wigner matrix. Let $w_{N}^{X}(x)$ be the rectangular Young diagram constructed from the eigenvalues of $X_{N}$ and $\hat{X}_{N}$. Note that $w_{N}^{X}(x)$ is a random function. We are interested in the limit behaviour of $w_{N}^{X}(x)$ as $N \rightarrow \infty$.

Let

$$
\Omega(x)= \begin{cases}\frac{2}{\pi}\left(x \arcsin \left(\frac{x}{2}\right)+\sqrt{4-x^{2}}\right), & |x| \leq 2, \\ |x|, & |x| \geq 2\end{cases}
$$

be the Vershik-Kerov-Logan-Shepp curve (see [12] and [10]).

Theorem 1. As $N \rightarrow \infty$, we have

$$
\lim _{n \rightarrow \infty} \sup _{x \in \mathbb{R}}\left|\frac{1}{\sqrt{N}} w_{N}^{X}(x \sqrt{N})-\Omega(x)\right|=0, \quad \text { in probability. }
$$




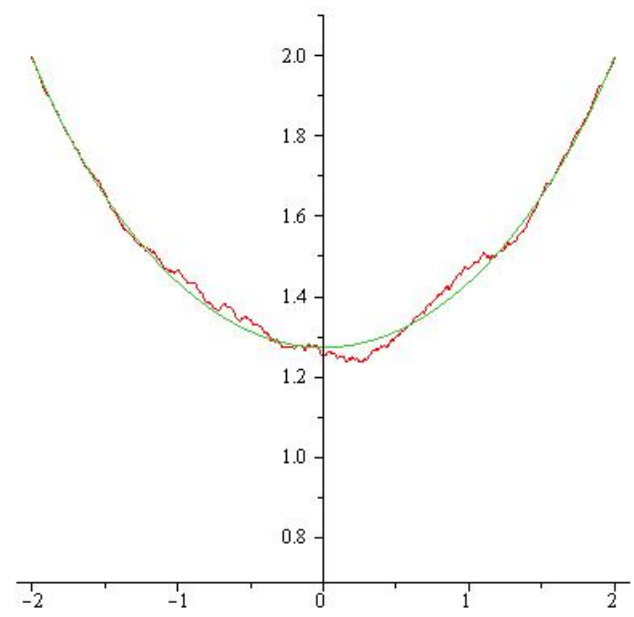

Figure 2: The rectangular Young diagram $w_{N}(x)$ and $\Omega(x)$ for $N=1100$

Interlacing sequences arise naturally in several areas. They provide a useful parametrization of Young diagrams (see [9, ,4]). They also appear as roots of two consecutive orthogonal polynomials (see [6]). A more general notion of interlacing measures was studied in [8].

For the first time the curve $\Omega(x)$ appeared from a representation theoretic problem. This curve is the limit shape of the random Young diagram distributed according to the Plancherel measure (see [12], 10], [13], and [4, Section 5] for more details). Then it was found that $\Omega(x)$ is a scaling limit for separation of roots of orthogonal polynomials (see [6]). This curve also arises as the scaling limit in the evolution of continuous Young diagrams (see [7]) and in random matrix theory. Let us formulate Kerov's result from 6] related to random matrix theory.

Let $h_{N} \subset \mathbb{R}^{N}$ be a random hyperplane such that $0 \in h_{N}$ and the normal vector to $h_{N}$ is uniformly distributed on the unit sphere. Let $p$ be the projection operator to $h_{N}$. Regard $X_{N}$ as an operator in $\mathbb{R}^{N}$ and consider the operator $p X_{N} p$ in $h_{N}$. The eigenvalues of $X_{N}$ and $p X_{N} p$ interlace. Let us construct the rectangular Young diagram $\tilde{w}_{N}$ from these eigenvalues as above.

Theorem ([6], Th. 3.6). As $N \rightarrow \infty$, we have

$$
\lim _{n \rightarrow \infty} \frac{1}{\sqrt{N}} \mathbf{E} \tilde{w}_{N}(x \sqrt{N})=\Omega(x)
$$

and the limit is uniform on $x \in \mathbb{R}$.

Remark 1. In the context of Theorem 1 we consider the restriction to a fixed hyperplane while here the hyperplane is random. Another difference is that 
Theorem 1 establishes the convergence in probability while 1.2 gives only the convergence of mean.

Let us proceed to Wishart matrices. Let $M=M(N)$ be a sequence of positive integers such that

$$
\lim _{N \rightarrow \infty} \frac{M(N)}{N}=\alpha \geq 1
$$

Let $\mathcal{W}_{N}$ be a $N \times M(N)$ matrix with i.i.d. entries of mean zero and variance 1 , and such that

$$
\mathbf{E}\left|W_{N}(1,1)\right|^{k}<\infty, \quad \text { for all } k=1,2,3, \ldots .
$$

By $Y_{N}$ we denote the $N \times N$ Wishart matrix $Y_{N}=\mathcal{W}_{N} \mathcal{W}_{N}^{t}$. Let $w_{N}^{Y}(x)$ be the rectangular Young diagram which is defined by the interlacing eigenvalues of $Y_{N}$ and $\hat{Y}_{N}$.

Let us define a continuous function $\Omega_{\alpha}: \mathbb{R} \rightarrow \mathbb{R}$. Set

$\Omega_{\alpha}^{\prime \prime}(x)=\frac{x+(\alpha-1)}{\pi x \sqrt{4 \alpha-(x-(\alpha+1))^{2}}}, \quad x \in[(\alpha+1)-2 \sqrt{\alpha} ;(\alpha+1)+2 \sqrt{\alpha}]$,

and

$$
\Omega_{\alpha}(x)=|x-\alpha|, \quad x \in(-\infty ;(\alpha+1)-2 \sqrt{\alpha}] \cup[(\alpha+1)+2 \sqrt{\alpha} ; \infty) .
$$

These formulas determine the function $\Omega_{\alpha}(x)$ uniquely.

Theorem 2. As $N \rightarrow \infty$, we have

$$
\lim _{n \rightarrow \infty} \sup _{x \in \mathbb{R}}\left|\frac{1}{N} w_{N}^{Y}(x N)-\Omega_{\alpha}(x)\right|=0, \quad \text { in probability. }
$$

Remark 2. The limit shapes $\Omega_{\alpha}(x)$ are closely connected with Biane's limit shapes (see [2]). This connection is described in Section 5.

This paper is organized as follows. In Section 2 we give some preliminaries. In Section 3 we prove Theorem 1. In Section 4 we prove Theorem 2. In Section 5 we describe a link between these limit shapes and semicircle and Marchenko-Pastur limit laws.

\section{Acknowledgments.}

The author is grateful to G. Olshanski and A. Borodin for valuable discussions. The author is grateful to V. Gorin and L. Petrov for valuable comments. The author was partially supported by Simons FoundationIUM scholarship, by Moebius Foundation scholarship, and by RFBR-CNRS grants 10-01-93114 and 11-01-93105. 


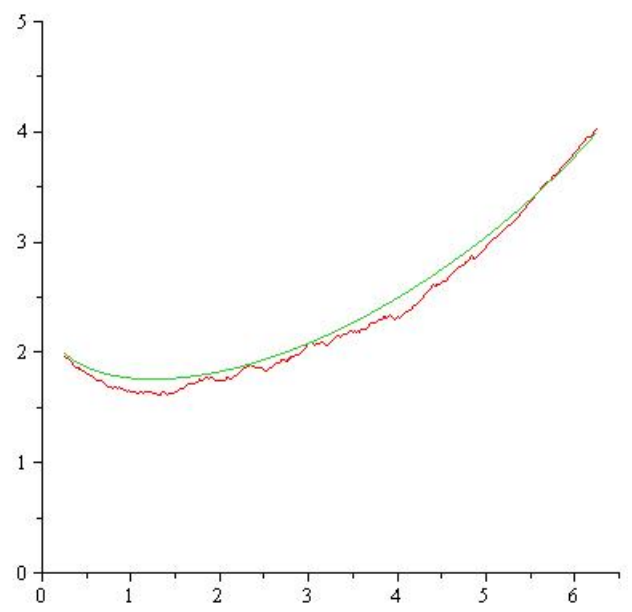

Figure 3: The rectangular Young diagram $w_{N}^{Y}(x)$ and $\Omega_{\alpha}(x)$ for $\alpha=2.25$ and $N=400$

\section{Continual Young diagrams}

A continual Young diagram (see [5]) is a function $w(x)$ on $\mathbb{R}$ such that

1) $\left|w\left(x_{1}\right)-w\left(x_{2}\right)\right| \leq\left|x_{1}-x_{2}\right|$ for any $x_{1}, x_{2} \in \mathbb{R}$.

2) There exists a point $x_{0} \in \mathbb{R}$, called the center of $w$, such that $w(x)=$ $\left|x-x_{0}\right|$ when $|x|$ is large enough.

The set of all continual Young diagrams is denoted by $\mathcal{D}$. For any $w \in \mathcal{D}$ we define a function $\sigma(x)$

$$
\sigma(x)=\frac{1}{2}(w(x)-|x|) .
$$

Since $\sigma(x)$ satisfies the Lipschitz condition 1), its derivative $\sigma^{\prime}(x)$ exists almost everywhere and satisfies $\left|\sigma^{\prime}(x)\right| \leq 1$. Note that $\sigma^{\prime}(x)$ is compactly supported. The function $w(x)$ is uniquely determined by $\sigma^{\prime}(x)$. Moreover, $w(x)$ is uniquely determined by the second derivative $\sigma^{\prime \prime}(x)$, which is understood in the sense of distribution theory.

Let us define the function $\tilde{p}_{k}: \mathcal{D} \rightarrow \mathbb{R}, k \in \mathbb{N}$, by setting

$$
\tilde{p}_{k}(w)=-k \int_{-\infty}^{\infty} x^{k-1} \sigma^{\prime}(x) d x=\int_{-\infty}^{\infty} x^{k} \sigma^{\prime \prime}(x) d x .
$$

It is easy to see that for the rectangular Young diagram $w^{\left\{x_{i}\right\},\left\{y_{j}\right\}}(x)$ (see Introduction) we have

$$
\sigma^{\prime \prime}(x)=\sum_{i=1}^{n} \delta\left(x-x_{i}\right)-\sum_{j=1}^{n-1} \delta\left(x-y_{j}\right)-\delta(x) .
$$


We will need the following fact.

Lemma 2.1 ([4], Lemma 5.7). Let $\mathcal{F}([a ; b])$ be the set of all real-valued functions $f(x)$ supported on the interval $[a, b] \in \mathbb{R}$ and satisfying the Lipschitz condition $\left|f\left(x_{1}\right)-f\left(x_{2}\right)\right| \leq\left|x_{1}-x_{2}\right|$.

On the set $\mathcal{F}([a ; b])$, the weak topology defined by the functionals

$$
f(x) \rightarrow \int_{x \in[a ; b]} f(x) x^{k} d x, \quad k=0,1,2, \ldots,
$$

coincides with the uniform topology.

\section{Proof of Theorem 1}

Lemma 3.1. Let $w_{N}^{X}$ be the (random) rectangular Young diagram defined by the eigenvalues of Wigner matrices $X_{N}$ and $\hat{X}_{N}$ (see Introduction). Then

$$
\frac{\tilde{p}_{k}\left(w_{N}^{X}\right)}{N^{k / 2}} \underset{N \rightarrow \infty}{\longrightarrow}\left\{\begin{array}{l}
0, \quad k \text { is odd, } \\
\frac{k !}{(k / 2) !(k / 2) !}, \quad k \text { is even, }
\end{array}\right.
$$

where the convergence is in probability.

Proof. The proof of this Lemma closely follows the proof of Wigner's theorem (see, e.g., [1, Section 2.1]) and is based on the well-known moment method.

Let $\left\{\lambda_{i}^{N}\right\}_{i=1}^{N}$ be the eigenvalues of $X_{N}$ and let $\left\{\lambda_{i}^{N-1}\right\}_{i=1}^{N-1}$ be the eigenvalues of $\hat{X}_{N}$. We have

$\tilde{p}_{k}\left(w_{N}^{X}\right)=\int_{x \in \mathbb{R}} x^{k}\left(\sum_{i=1}^{N} \delta\left(\lambda_{i}^{N}\right)-\sum_{j=1}^{N-1} \delta\left(\lambda_{j}^{N-1}\right)\right) d x=\operatorname{tr}\left(X_{N}^{k}\right)-\operatorname{tr}\left(X_{N-1}^{k}\right)$.

Let $\mathbf{i}_{N}=\left(i_{1}, i_{2}, \ldots, i_{k}\right)$ range over the set of multi-indices such that $1 \leq$ $i_{1}, i_{2}, \ldots, i_{k} \leq N$. Likewise, let $\mathbf{i}_{N-1}=\left(i_{1}, i_{2}, \ldots, i_{k}\right)$ range over the set of multi-indices such that $1 \leq i_{1}, i_{2}, \ldots, i_{k} \leq N-1$. Then

$$
\begin{aligned}
& \operatorname{tr}\left(X_{N}^{k}\right)-\operatorname{tr}\left(X_{N-1}^{k}\right)=\sum_{\mathbf{i}_{N}} X_{N}\left(i_{1}, i_{2}\right) X_{N}\left(i_{2}, i_{3}\right) \ldots X\left(i_{k}, i_{1}\right) \\
& -\sum_{\mathbf{i}_{N-1}} X_{N}\left(i_{1}, i_{2}\right) X_{N}\left(i_{2}, i_{3}\right) \ldots X\left(i_{k}, i_{1}\right)=\sum_{\mathbf{i}_{N}: N \in \mathbf{i}_{N}} X_{N}\left(i_{1}, i_{2}\right) X_{N}\left(i_{2}, i_{3}\right) \ldots X\left(i_{k}, i_{1}\right),
\end{aligned}
$$

where the last sum is taken over indices $\mathbf{i}_{N}=\left(i_{1}, i_{2}, \ldots, i_{k}\right)$ such that there exists $r, 1 \leq r \leq k$, satisfying $i_{r}=N$.

First, we compute

$$
\mathbf{E}\left(\sum_{\mathbf{i}_{N}: N \in \mathbf{i}_{N}} X_{N}\left(i_{1}, i_{2}\right) X_{N}\left(i_{2}, i_{3}\right) \ldots X\left(i_{k}, i_{1}\right)\right) .
$$


This sum can be written as a sum of terms corresponding to suitably defined graphs that are in their turn associated to words. Suppose $k$ is odd; then the same estimates as in [1, Lemma 2.1.6] show that the contribution to the degree $n^{k}$ is equal to 0 .

Suppose $k$ is even; then the main contribution is given by the so called Wigner words (see [1, Def. 2.1.10]). The number of Wigner words is equal to $\frac{k !}{(k / 2+1) !(k / 2) !}$. The only difference of our case with the case of Wigner's theorem is that one vertex of a graph should be labeled by the special symbol $N$. This condition gives an extra factor $(k / 2+1)$. Thus, we obtain

$$
\begin{aligned}
\lim _{N \rightarrow \infty} N^{-k / 2} \mathbf{E}\left(\sum_{\mathbf{i}_{N}: N \in \mathbf{i}_{N}} X_{N}\left(i_{1}, i_{2}\right)\right. & \left.X_{N}\left(i_{2}, i_{3}\right) \ldots X\left(i_{k}, i_{1}\right)\right) \\
& = \begin{cases}0, & k \text { is odd }, \\
\frac{k !}{(k / 2) !(k / 2) !}, & k \text { is even. }\end{cases}
\end{aligned}
$$

Secondly, we have

$$
\lim _{N \rightarrow \infty}\left(N^{-k / 2} \sum_{\mathbf{i}_{N}: N \in \mathbf{i}_{N}} X_{N}\left(i_{1}, i_{2}\right) X_{N}\left(i_{2}, i_{3}\right) \ldots X\left(i_{k}, i_{1}\right)\right)^{2}=0 .
$$

Indeed, this equality can be proved in the same way as in [1, Lemma 2.1.7].

From $(3.2)$ and $(3.3)$ it follows that $\tilde{p}_{k}$ converges to the right-hand side of (3.1) in $L^{2}$ and, consequently, in probability.

Lemma 3.2 (4] Prop. 5.3). We have

$$
\tilde{p}_{k}(\Omega)=\left\{\begin{array}{l}
0, \quad k \text { is odd }, \\
\frac{k !}{(k / 2) !(k / 2) !}, \quad k \text { is even. }
\end{array}\right.
$$

Lemma 3.3. There exists an interval $[-B ; B]$ such that the probability that the inequalities

$$
-B<\lambda_{N}^{N} \leq \cdots \leq \lambda_{1}^{N}<B
$$

hold tends to 1 as $N \rightarrow \infty$.

Proof. This is a well-known fact from random matrix theory, see, e.g. [1].

Let $z_{N}$ be the center of the rectangular Young diagram $w_{N}^{X}$. It is clear that

$$
\lim _{N \rightarrow \infty} \frac{z_{N}}{\sqrt{N}}=\lim _{N \rightarrow \infty} \frac{X(N, N)}{\sqrt{N}}=0, \quad \text { in probability. }
$$


This equality and Lemma 3.3 imply the convergence of $w_{N}^{X}$ to $\Omega(x)$ outside the interval $[-B ; B]$.

From Lemmas 3.1 and 3.2 for any $k \in \mathbb{N}$ we have

$$
\lim _{N \rightarrow \infty} \frac{\tilde{p}_{k}\left(w_{N}^{X}\right)}{N^{k / 2}}=\tilde{p}_{k}(\Omega), \quad \text { in probability. }
$$

The convergence of $w_{N}^{X}$ to $\Omega(x)$ inside the interval $[-B ; B]$ follows from (3.4) and Lemma 2.1.

\section{Proof of Theorem 2}

Lemma 4.1. Let $w_{N}^{Y}$ be the (random) rectangular Young diagram defined by the eigenvalues of Wishart matrices $Y_{N}$ and $\hat{Y}_{N}$ (see Introduction). Then the following limit exists

$$
\frac{\tilde{p}_{k}\left(w_{N}^{Y}\right)}{N^{k}} \underset{N \rightarrow \infty}{\longrightarrow} m_{k}, \quad \text { in probability. }
$$

The generating function of $m_{k}$ is given by the equation

$$
G_{\alpha}(z):=1+\sum_{k=1}^{\infty} m_{k} z^{k}=\frac{1}{2}\left(1+\frac{(\alpha-1) z+1}{\sqrt{(\alpha-1)^{2} z^{2}-2(\alpha+1) z+1}}\right) .
$$

Proof. The proof of this Lemma is based on moment method and follows that given in [1, Exercise 2.1.18].

Let $\mathbf{i}_{N}=\left(i_{1}, i_{2}, \ldots, i_{N}\right), \mathbf{j}_{N}=\left(j_{1}, j_{2}, \ldots, j_{N}\right)$ range over the set of multiindices such that $1 \leq i_{1}, i_{2}, \ldots, i_{N} \leq N, 1 \leq j_{1}, j_{2}, \ldots, j_{N} \leq M(N)$. We have

$$
\begin{aligned}
& \tilde{p}_{k}\left(w_{N}^{Y}\right)=\operatorname{tr}\left(Y_{N}^{k}\right)-\operatorname{tr}\left(Y_{N-1}^{k}\right)=\sum_{\mathbf{i}_{N}: N \in \mathbf{i}_{N}} Y_{N}\left(i_{1}, i_{2}\right) Y_{N}\left(i_{2}, i_{3}\right) \ldots Y_{N}\left(i_{N}, i_{1}\right) \\
= & \sum_{\mathbf{i}_{N}, \mathbf{j}_{N}: N \in \mathbf{i}_{N}} \mathcal{W}_{N}\left(i_{1}, j_{1}\right) \mathcal{W}_{N}\left(i_{2}, j_{1}\right) \mathcal{W}_{N}\left(i_{2}, j_{1}\right) \mathcal{W}_{N}\left(i_{2}, j_{2}\right) \ldots \mathcal{W}_{N}\left(i_{k}, j_{k}\right) \mathcal{W}_{N}\left(i_{1}, j_{k}\right),
\end{aligned}
$$

where the condition $N \in \mathbf{i}_{N}$ means, as before, that there exists $r$ such that $i_{r}=N$.

The limit relation

$$
\lim _{N \rightarrow \infty} \frac{\mathbf{E}\left(\operatorname{tr}\left(Y_{N}^{k}\right)-\operatorname{tr}\left(Y_{N-1}^{k}\right)\right)^{2}}{N^{2 k}}=0
$$

can be proved in the same way as in [1, Section 2.1]. Thus, the only thing we need is to compute the leading term in

$\mathbf{E}\left(\sum_{\mathbf{i}_{N}, \mathbf{j}_{N}: N \in \mathbf{i}_{N}} \mathcal{W}_{N}\left(i_{1}, j_{1}\right) \mathcal{W}_{N}\left(i_{2}, j_{1}\right) \mathcal{W}_{N}\left(i_{2}, j_{1}\right) \mathcal{W}_{N}\left(i_{2}, j_{2}\right) \ldots \mathcal{W}_{N}\left(i_{k}, j_{k}\right) \mathcal{W}_{N}\left(i_{1}, j_{k}\right)\right)$. 
Let us recall that a Dyck path $\mathcal{D}_{l}$ of length $2 l$ is an integer-valued sequence $\left\{S_{n}\right\}_{0 \leq n \leq 2 l}$ such that $S_{0}=0, S_{2 l}=0,|S(i)-S(i-1)|=1$ for $1 \leq i \leq 2 l$, and $S(i) \geq 0$ for $0 \leq i \leq 2 l$. Let $a\left(\mathcal{D}_{l}\right)$ be the number of indices $i$ such that $S(i)-S(i-1)=-1$ and $S(i-1)$ is odd and let $b\left(\mathcal{D}_{l}\right)$ be the number of indices $i$ such that $S(i)-S(i-1)=-1$ and $S(i-1)$ is even. We have $a\left(\mathcal{D}_{l}\right)+b\left(\mathcal{D}_{l}\right)=l$.

There is a bijective correspondence between Wigner words and Dyck paths (see [1, Section 2.1]). It is easy to see that the main contribution to 4.2 has order $N^{k}$ and is given by Wigner words of length $2 k$ or, equivalently, by Dyck paths of length $2 k$. If the sequence $\left(i_{1}, j_{1}, i_{2}, j_{2}, \ldots, i_{k}, j_{k}\right)$ is a Wigner word then $a\left(\mathcal{D}_{k}\right)$ is equal to the number of distinct $j$-indices in the tuple and $b\left(\mathcal{D}_{k}\right)+1$ is equal to the number of distinct $i$-indices in the tuple, where $\mathcal{D}_{k}$ is the corresponding Dyck path. Therefore, each word gives a contribution $\left(b\left(\mathcal{D}_{k}\right)+1\right) \alpha^{a\left(\mathcal{D}_{k}\right)}$ and we obtain

$$
m_{k}=\sum_{\text {all } \mathcal{D}_{k}}\left(b\left(\mathcal{D}_{k}\right)+1\right) \alpha^{a\left(\mathcal{D}_{k}\right)} .
$$

Let $\beta$ be a formal variable, and let

$$
\begin{aligned}
d_{r}:=\sum_{\text {all } \mathcal{D}_{r}} \beta^{r-a\left(\mathcal{D}_{r}\right)} \alpha^{a\left(\mathcal{D}_{r}\right)}, & r \geq 1, & d_{0}=1 ; \\
e_{r}:=\sum_{\text {all } \mathcal{D}_{r}} \beta^{a\left(\mathcal{D}_{r}\right)} \alpha^{r-a\left(\mathcal{D}_{r}\right)}, & r \geq 1, & e_{0}=1 .
\end{aligned}
$$

Considering the moment of the first return of Dyck path $D_{r}$ to zero we obtain

$$
d_{r}=\alpha \sum_{j=1}^{r} d_{r-j} e_{j-1}, \quad e_{r}=\beta \sum_{j=1}^{r} e_{r-j} d_{j-1} .
$$

Hence,

$$
d_{r}=\frac{\alpha}{\beta} e_{r}, \quad r \geq 1
$$

We obtain

$$
d_{r}=\beta \sum_{j=2}^{r} d_{r-j} d_{j-1}+\alpha d_{r-1} .
$$

Let $d(z)$ be the generating function of $\left\{d_{r}\right\}$ :

$$
d(z):=1+\sum_{r=1}^{\infty} d_{r} z^{r} .
$$

Using (4.4), we get

$$
d(z)=1+\beta z d(z)^{2}+(\alpha-\beta) z d(z) .
$$


Solving this equation and choosing the sign from the condition $d(0)=1$, we obtain

$$
d(z)=\frac{1-(\alpha-\beta) z-\sqrt{((\alpha-\beta) z-1)^{2}-4 \beta z}}{2 \beta z} .
$$

From 4.3, we get

$$
1+\sum_{k=1}^{\infty} m_{k} z^{k}=\left.\frac{\partial}{\partial \beta}(\beta d(z))\right|_{\beta=1} .
$$

This completes the proof of Lemma 4.1.

For $\alpha>1$ let $\Omega_{\alpha}(x)$ be defined by the formula

$\Omega_{\alpha}(x):=\left\{\begin{array}{l}\frac{1}{\pi}\left((2 \alpha-x) \arcsin \left(\frac{\alpha+1-x}{2 \sqrt{\alpha}}\right)-\arctan \left(\frac{(\alpha-1)^{2}-x(\alpha+1)}{(\alpha-1) \sqrt{(x-\alpha)^{2}+2 \alpha+2 x-1}}\right)\right. \\ \left.+\sqrt{2 \alpha+2 x-1-(x-\alpha)^{2}}\right), \quad \text { for } x \in[\alpha+1-2 \sqrt{\alpha} ; \alpha+1+2 \sqrt{\alpha}] \\ |x-\alpha|, \quad \text { for } x \in(-\infty ;(\alpha+1)-2 \sqrt{\alpha}] \cup[(\alpha+1)+2 \sqrt{\alpha} ; \infty),\end{array}\right.$

and for $\alpha=1$ let

$\Omega_{1}(x):=\left\{\begin{array}{l}\frac{1}{\pi}\left((x-2) \arcsin \left(\frac{1}{2} x-1\right)-\sqrt{4-(x-2)^{2}}\right)+\frac{x}{2}, \\ |x-1|, \quad \text { for } x \in(-\infty ; 0] \cup[2 ; \infty) .\end{array}\right.$

It is readily seen that for $\alpha \geq 1, \Omega_{\alpha}(x)$ is a continual Young diagram with the center $\alpha$.

Lemma 4.2. The generating function of $\left\{\tilde{p}_{k}\left(\Omega_{\alpha}\right)\right\}$ is given by the formula

$$
1+\sum_{k=1}^{\infty} \tilde{p}_{k}\left(\Omega_{\alpha}\right) z^{k}=\frac{1}{2}\left(1+\frac{(\alpha-1) z+1}{\sqrt{(\alpha-1)^{2} z^{2}-2(\alpha+1) z+1}}\right) .
$$

Proof. We recall that

$$
\tilde{p}_{k}\left(\Omega_{\alpha}\right)=\int_{\mathbb{R}} x^{k} \frac{\Omega_{\alpha}^{\prime \prime}(x)}{2} d x, \quad k \in \mathbb{N} .
$$

It can be verified by a direct computation that for $\alpha \geq 1$

$\Omega_{\alpha}^{\prime \prime}(x)=\left\{\begin{array}{l}\frac{x+(\alpha-1)}{\pi x \sqrt{4 \alpha-(x-(\alpha+1))^{2}}}, \quad x \in[\alpha+1-2 \sqrt{\alpha} ; \alpha+1+2 \sqrt{\alpha}], \\ 0, \quad x \neq[\alpha+1-2 \sqrt{\alpha} ; \alpha+1+2 \sqrt{\alpha}] .\end{array}\right.$

Using the technique of Stieltjes transform (see, e.g., [1, Section 2.4]) we obtain the statement of the Lemma. 
Let $z_{N}^{Y}$ be the center of the diagram $w_{N}^{Y}$. Note that

$$
\frac{z_{N}^{Y}}{N}=\frac{Y_{N}(N, N)}{N}=\frac{\sum_{j=1}^{N} \mathcal{W}(N, j) \mathcal{W}(j, N)}{N} \underset{N \rightarrow \infty}{\longrightarrow} \alpha, \quad \text { in probability. }
$$

This fact implies that the center of $\frac{1}{N} w_{N}^{Y}(N x)$ converges to the center of $\Omega(\alpha)$. From Lemmas 4.1 and 4.2 we have

$$
\frac{\tilde{p}_{k}\left(w_{N}^{Y}\right)}{N^{k}} \underset{N \rightarrow \infty}{\longrightarrow} \tilde{p}_{k}\left(\Omega_{\alpha}\right), \quad \text { in probability. }
$$

Also it is known that the spectrum of Wishart matrix is supported by a fixed compact interval with probability close to 1 . Theorem 2 follows from these facts in the same way as in the proof of Theorem 1 .

\section{Connection with semicircle and Marchenko-Pastur laws}

In this Section we briefly describe a connection between limiting shapes $\Omega$, $\Omega_{\alpha}$ and well-known semicircle and Marchenko-Pastur distributions, respectively.

For an interval $I$ by $\mathcal{M}(I)$ we denote the set of probability measures which are supported by $I$. For a measure $\mu \in \mathcal{M}(I)$ let

$$
\mu_{k}:=\int_{I} x^{k} d \mu, \quad k \in \mathbb{N} .
$$

By $\mathcal{D}(I)$ we denote the set of continual Young diagrams such that $\sigma^{\prime \prime}(x)$ is supported by $I$.

Lemma 5.1. There is a bijective correspondence $\mu \rightarrow w$ between $\mathcal{M}(I)$ and $\mathcal{D}(I)$. It is characterized by the relation

$$
1+\sum_{k=1}^{\infty} \mu_{k} z^{k}=\exp \left(\sum_{k=1}^{\infty} \frac{\tilde{p}_{k}(w)}{k} z^{k}\right)
$$

Proof. See [5], 8].

The measure $\mu$ is called the transition measure of $w$. For more details about this correspondence, see [5], 4, Section 8].

A direct computation of the left- and right-hand sides of (5.1) leads to the following Proposition.

Proposition 5.1. a) The semicircle law is the transition measure of $\Omega$.

b) The Marchenko-Pastur distribution with parameter $\alpha$ is the transition measure of $\Omega_{\alpha}$. 
The item a) was first noted in [5].

In [2] Biane introduced a family of curves which appeared as scaling limits in some problem of asymptotic representation theory. As shown in [11, the transition measure of every Biane's curve coincide with a MarchenkoPastur distribution, within a homothetic transform. Thus the Biane's curves and the curves $\Omega_{\alpha}$ are closely related.

Remark 3. Let $\mathbf{x}_{n}=\left(x_{1}, x_{2}, \ldots, x_{n}\right)$ be a sequence of real numbers and suppose that for every $n$ the sequences $\mathbf{x}_{n}$ and $\mathbf{x}_{n-1}$ interlace. Let $\mu$ be a probability measure on $\mathbb{R}$ and let $\delta(x)$ be the Dirac measure at $x \in \mathbb{R}$. Assume that

$$
\frac{1}{n} \sum_{i=1}^{n} \delta\left(x_{i}\right) \underset{n \rightarrow \infty}{\longrightarrow} \mu, \quad \text { in the weak topology. }
$$

It was shown in [6] that there are sequences with different limiting measures

$\mu$ but with the same scaling limit of $w^{\mathbf{x}_{n}, \mathbf{x}_{n-1}}$. Therefore, the convergence of $\frac{1}{n} \sum_{i=1}^{n} \delta\left(x_{i}\right)$ to the measure $\mu$ does not imply the convergence of $w^{\mathbf{x}_{n}, \mathbf{x}_{n-1}}$ to the continual Young diagram with the transition measure $\mu$.

\section{References}

[1] G. W. Anderson, A. Guionnet, and O. Zeitouni, An introduction to random matrices, Cambridge University Press, 2010.

[2] P. Biane, Approximate factorization and concentration for characters of symmetric groups, Inter. Math. Res. Notices 2001 (2001), no. 4, 179-192.

[3] R. A. Horn and C. R. Johnson, Matrix Analysis, Cambridge University Press, New York, 1985.

[4] V. Ivanov and G. Olshanski. Kerov's central limit theorem for the Plancherel measure on Young diagrams. In Symmetric Functions 2001: Surveys of Developments and Perspectives, volume 74 of NATO Science Series II. Mathematics, Physics and Chemistry, pages 93-151, 2002.

[5] S. Kerov, Transition Probabilities of Continual Young Diagrams and Markov Moment Problem, Funktsion. Anal. i Prilozhen. 27 (1993), no. 2, 32-49; English translation: Funct. Anal. Appl. 27 (1993), 104-117.

[6] S. Kerov, Asymptotics of the separation of roots of orthogonal polynomials, St. Petersburg Math. J. 5 (1994), 925-941.

[7] S. Kerov, The differential model of growth of Young diagrams, Proc. St. Petersburg Math. Soc. 4 (1996), 167-194. 
[8] S. Kerov, Interlacing measures, In: Kirillov's seminar on representation theory (G. Olshanski, ed.), Amer. Math. Soc., Providence, RI, 1998, pp. $35-83$.

[9] S. V. Kerov, Anisotropic Young diagrams and Jack symmetric functions, Funktsional. Anal. i Prilozhen. 34 (2000), no. 1, 51-64 (Russian); English translation: Funct. Anal. Appl. 34 (2000), 41-51

[10] F. Logan and L. A. Shepp, A variational problem for random Young tableaux, Advances in Math. 26 (1977), 206-222.

[11] P.L. Méliot. Kerov's central limit theorem for Schur-Weyl measures of parameter 1/2, arXiv:1009.4034.

[12] A. M. Vershik and S. V. Kerov, Asymptotics of the Plancherel measure of the symmetric group and the limiting form of Young tableaux, Doklady AN SSSR 233 (1977), no. 6, 1024-1027; English translation: Soviet Mathematics Doklady 18 (1977), 527-531.

[13] A. M. Vershik, S. V. Kerov, Asymptotic theory of characters of the symmetric group, Function. Anal. i Prilozhen. 15 (1981), no. 4, 15-27; English translation: Funct. Anal. Appl. 15 (1985), 246-255. 